\title{
Stable forking and imaginaries
}

\author{
Enrique Casanovas*and Joris Potier \\ University of Barcelona
}

August 7, 2015

\begin{abstract}
We prove that a theory $T$ has stable forking if and only if $T^{\mathrm{eq}}$ has stable forking.
\end{abstract}

\section{Introduction}

We follow the standard conventions, $T$ is a complete theory of language $L$ and $\mathfrak{C}$ is its monster model. A formula $\varphi(x, y)$ (where $x, y$ are disjoint tuples of variables) is stable if there are not $\left(a_{i} \mid i<\omega\right)$ and $\left(b_{i} \mid i<\omega\right)$ such that $\models \varphi\left(a_{i}, b_{j}\right)$ if and only if $i<j$.

It is said that $T$ has stable forking if whenever a type $p(x) \in S(B)$ forks over some subset $A \subseteq B$, there is some stable formula $\varphi(x, y) \in L$ and some tuple $b \in B$ such that $\varphi(x, b) \in p(x)$ and $\varphi(x, b)$ forks over $A$. The stable forking conjecture says that every simple theory has stable forking.

Remark 1.1 1. If $\varphi(x, y)$ is a boolean combination of stable formulas $\varphi_{i}\left(x_{i}, y_{i}\right)$ (where $x_{i} \subseteq x, y_{i} \subseteq y$ and $\left.x_{i} \cap y_{j}=\emptyset\right)$ then $\varphi(x, y)$ is stable.

2. If $\varphi(x, y)$ is stable, then $\varphi^{-1}(y, x)=\varphi(x, y)$ (the same formula with the role of $x, y$ interchanged) is stable.

3. If $\varphi(x, a) \equiv \psi(x, b)$ and $\psi(x, z)$ is stable, then for some $\mu(y) \in \operatorname{tp}(a), \varphi(x, y) \wedge \mu(y)$ is stable.

4. In order to check that $T$ has stable forking, it is enough to consider types over models.

5. If $\varphi(x, y)$ is stable and $p(x) \in S_{\varphi}(M)$, then $p(x)$ is definable by a boolean combination of formulas $\varphi(m, y)$ for some tuples $m \in M$. The canonical base of $p(x)$ is an imaginary e, the canonical parameter of (any) definition of $p(x)$ over $M$. If $A \subseteq M$, then $p(x)$ forks over $A$ if and only if $e \notin \operatorname{acl}^{\mathrm{eq}}(A)$. For any model $N \supseteq M, p(x)$ has a unique e-definable extension $p^{\prime}(x) \in S_{\varphi}(N)$.

Proof: For 1, 2 and 5 see chapters 6 and 8 of [1] or chapter 1 of [7]. For 3 and 4 see [4].

If $\varphi(x, y)$ is stable and $p(x)$ is a $\varphi$-type over a model $M$, then $p$ does not fork over its canonical base, an imaginary $e \in \mathrm{dcl}^{\mathrm{eq}}(M)$. Therefore, if $T$ has stable forking then for every

*Partially supported by the Spanish government grant MTM 2011-26840 and the Catalan government grant 2014SGR-437. 
type $p(x) \in S(M)$ there is a subset $A \subseteq M$ such that $|A| \leq|T|$ and $p$ does not fork over $A$. This means that if $T$ has stable forking, then $T$ is simple. More generally, A. Chernikov has shown (see Proposition 4.14 in [2]) that if $T$ has simple forking (meaning that forking is always witnessed by a simple formula) then $T$ is simple.

There is not much progress on the stable forking conjecture. B. Kim proved in 3 that simple one-based theories with elimination of hyperimagnaries have stable forking. A. Peretz in [6] proved that types of SU-rank two in $\omega$-categorical supersimple theories have stable forking. In [5] D. Palacín and F.O. Wagner have shown that supersimple CMtrivial $\omega$-categorical theories have stable forking. Finally, let us mention that stable forking implies weak elimination of hyperimaginaries (see [4]).

We will need the following lemma on algebraic quantification of a stable formula:

Lemma 1.2 If $\varphi(x, y) \in L$ is stable and $\theta(v, x) \vdash \exists^{=n} x \theta(v, x)$, then $\psi(v, y)=\exists x(\theta(v, x) \wedge$ $\varphi(x, y))$ is stable.

Proof: Assume $\models \psi\left(a_{i}, b_{j}\right)$ iff $i<j$. For each $i<\omega, \models \exists x \theta\left(a_{i}, x\right)$ and hence there are different $c_{i}^{1}, \ldots, c_{i}^{n}$ such that $\models \theta\left(a_{i}, c_{i}^{k}\right)$ for all $k=1, \ldots, n$. Whenever $i<j<\omega$ choose some $k_{i j}$ such that $1 \leq k_{i j} \leq n$ and $\models \theta\left(a_{i}, c_{i}^{k_{i j}}\right) \wedge \varphi\left(c_{i}^{k_{i j}}, b_{j}\right)$. By Ramsey's theorem, for some infinite $I \subseteq \omega$ there is some $k$ such that $1 \leq k \leq n$ and $\models \theta\left(a_{i}, c_{i}^{k}\right) \wedge \varphi\left(c_{i}^{k}, b_{j}\right)$ for all $i, j \in I$ such that $i<j$. Then for $i, j \in I: \models \varphi\left(c_{i}^{k}, b_{j}\right)$ iff $i<j$, which shows that $\varphi(x, y)$ is unstable.

The following remark is a stronger version of item 4 of Remark 1.1, with a similar proof. We won't use it in this article. The proof uses generalized $\varphi$-types (see chapter 6 of [1]). The main point is that nonforking is transitive for these types (if $\varphi$ is stable) and over models they coincide with ordinary $\varphi$-types. The generalized $\varphi$-type of $a$ over $A$ is the set of all formulas in $\operatorname{tp}(a / A)$ which are equivalent to boolean combinations of $\varphi$-formulas over the monster model.

Remark 1.3 If whenever a type $p(x) \in S(N)$ forks over an elementary submodel $M \subseteq N$, there is an instance of a stable formula in $p(x)$ witnessing forking over $M$, then $T$ has stable forking.

Proof: We can assume that $T$ is simple. Assume $A \subseteq B$ and $a \not_{A} B$. Choose a model $M \supseteq A$ such that $M \downarrow_{A} B a$ and note that $a \mathbb{X}_{M} B$. Now choose a model $N \supseteq M B$ such that $N \downarrow_{M B} a$ and note that $a \bigotimes_{M} N$. By the assumption, there is a stable formula $\varphi(x, y) \in L$ and some tuple $n \in N$ such that $\models \varphi(a, n)$ and $\varphi(x, n)$ forks over $M$. Let $p(x)$ be the $\varphi$-type of $a$ over $N$ and $q(x)$ the generalized $\varphi$-type of $a$ over $B$. Since $p(x)$ forks over $A$ but it does not fork over $B, q(x)$ forks over $A$. Hence there is some formula $\psi(x, z) \in L$ and some tuple $b \in B$ such that $\psi(x, b)$ is in $q(x)$ and forks over $A$. By item 3 of Remark 1.1, there is some formula $\mu(z) \in \operatorname{tp}(b)$ such that $\psi^{\prime}(x, z)=\psi(x, z) \wedge \mu(z)$ is stable. But $\models \psi^{\prime}(a, b)$ and $\psi^{\prime}(x, b)$ forks over $A$.

\section{Main result}

In this section $T$ is a simple theory, but since stable forking implies simplicity, in fact it is not necessary to add this assumption to the propositions below. Hence forking and dividing is the same thing in $T$.

Proposition 2.1 If $T$ has stable forking, then $T^{\mathrm{eq}}$ has stable forking over real parameters. 
Proof: Let $A \subseteq B \subseteq \mathfrak{C}$, let $e \in \mathfrak{C}^{\mathrm{eq}}$ be an imaginary of sort $E$ and assume $e \mathbb{L}_{A} B$. Choose a $|A|^{+}+\omega$-saturated model $M \supseteq B$ such that $e \in \mathrm{dcl}^{\mathrm{eq}}(M)$ and a representative $a$ of $e=a_{E}$ such that $a \downarrow_{e} M$. It follows that $a \bigsqcup_{A} B$. By assumption, there is some stable formula $\delta(x, y) \in L$ and some tuple $b \in B$ such that $\models \delta(a, b)$ and $\delta(x, b)$ forks over $A$. Consider the type $p(x)=\operatorname{tp}_{\delta}(a / M)$. It has a definition $d_{p} x \delta(x, y)$ which is a boolean combination of formulas of the form $\delta(m, y)$ for some tuples $m \in M$. The definition is, therefore, an instance of a stable formula. Note that $p(x)$ does not fork over $e$ and hence its canonical basis $d_{F} \in M^{\mathrm{eq}}$ is in $\operatorname{acl}^{\mathrm{eq}}(e)$. For some $\chi(w, y) \in L^{\mathrm{eq}}, \chi\left(d_{F}, y\right)$ defines $p(x)$. Since $d_{p} x \delta(x, y) \equiv \chi\left(d_{F}, y\right)$, by item 3 of Lemma 1.1 for some $\mu(w) \in \operatorname{tp}\left(d_{F}\right)$, the formula $\chi(w, y) \wedge \mu(y)$ is stable. Without loss of generality then $\chi(w, y)$ is stable. Note that since $\delta(x, b) \in p(x), \models \chi\left(d_{F}, b\right)$.

Claim 1: If $q(w)=\operatorname{tp}\left(d_{F}\right)$, then $q(w) \cup\{\chi(w, b)\}$ forks over $A$.

Proof: Assume not. We will prove that $\delta(x, b)$ does not divide over $A$, which is a contradiction. Let $\left(b_{i} \mid i<\omega\right)$ be an $A$-indiscernible sequence of tuples $b_{i} \equiv_{A} b$ and let us check that $\left\{\delta\left(x, b_{i}\right) \mid i<\omega\right\}$ is consistent. By the saturation of $M$, we may assume that $b_{i} \in M$ for all $i<\omega$. By our assumption in the proof, $q(w) \cup\left\{\chi\left(w, b_{i}\right) \mid i<\omega\right\}$ is consistent and hence we can find some realization $d_{F}^{\prime} \in M^{\text {eq }}$ of this set of formulas. Since $d_{F} \equiv d_{F}^{\prime}$, there is some sequence $\left(b_{i}^{\prime} \mid i<\omega\right)$ in $M^{\text {eq }}$ such that $d_{F}\left(b_{i}^{\prime} \mid i<\omega\right) \equiv d_{F}^{\prime}\left(b_{i} \mid i<\omega\right)$. Then $\models \chi\left(d_{F}, b_{i}^{\prime}\right)$ for every $i<\omega$, which implies $\delta\left(x, b_{i}^{\prime}\right) \in p(x)$ and $\models \delta\left(a, b_{i}^{\prime}\right)$ for all $i<\omega$. Since $\left\{\delta\left(x, b_{i}^{\prime}\right) \mid i<\omega\right\}$ is consistent, $\left\{\delta\left(x, b_{i}\right) \mid i<\omega\right\}$ is consistent too.

With Claim 1 we can now choose some $\mu(w) \in q(w)$ such that $\chi(w, b) \wedge \mu(w)$ forks over $A$. Note that $\chi^{\prime}(w, y)=\chi(w, y) \wedge \mu(w)$ is stable. Since $\chi^{\prime}(w, b)$ divides over $A$, this can be witnessed by an $A$-indiscernible sequence $\left(b_{i} \mid i<\omega\right)$ with $b_{i} \equiv_{A} b$ and some $k<\omega$ for which $\left\{\chi^{\prime}\left(w, b_{i}\right) \mid i<\omega\right\}$ is $k$-inconsistent. Since $d_{F} \in \operatorname{acl}^{\mathrm{eq}}(e)$, there is some formula $\theta(v, w) \in L^{\text {eq }}$ and some $n<\omega$ such that $\models \theta\left(e, d_{F}\right)$ and $\theta(v, w) \vdash \exists^{=n} w \theta(v, w)$. Let $\varphi(v, y)=\exists w\left(\theta(v, w) \wedge \chi^{\prime}(w, y)\right)$. By Lemma 1.2, $\varphi(v, y)$ is stable. Since $\models \varphi(e, b)$, it only remains to check that $\varphi(v, b)$ forks over $A$. This is done in the next claim.

Claim 2: $\varphi(v, b)$ divides over $A$ with respect to $l=n(k-1)+1$, witnessed by $\left(b_{i} \mid i<\omega\right)$. Proof: Otherwise $\left\{\varphi\left(v, b_{i}\right) \mid i<\omega\right\}$ is consistent and it is realized by some $e^{\prime} \in \mathfrak{C}^{\mathrm{eq}}$. For each $i<l$ choose some $d_{F}^{i}$ such that $\models \theta\left(e^{\prime}, d_{F}^{i}\right) \wedge \chi^{\prime}\left(d_{F}^{i}, b_{i}\right)$. The number of $d_{F}^{i}$ is $\leq n$ and therefore, by choice of $l$, the mapping $i \mapsto d_{F}^{i}$ has some fiber of cardinality $\geq k$. This shows that $\left\{\chi^{\prime}\left(w, b_{i}\right) \mid i<\omega\right\}$ is $k$-consistent, a contradiction with the choice of $k$.

Proposition 2.2 If $T^{\mathrm{eq}}$ has stable forking over real parameters, then $T^{\mathrm{eq}}$ has stable forking.

Proof: By item 4 of Remark 1.1, it is enough to consider types over models. Assume $e \mathbb{Z}_{A} M^{\mathrm{eq}}$, where $M \subseteq \mathfrak{C}$ is a model, $M^{\mathrm{eq}}=\mathrm{dcl}^{\mathrm{eq}}(M)$ is the corresponding imaginary model, $A \subseteq M^{\mathrm{eq}}$ and $e \in \mathfrak{C}^{\mathrm{eq}}$. Choose a set $A^{\prime}$ of representatives of the elements of $A$ such that $A^{\prime} \downarrow_{A} M e$. Then $e \mathbb{L}_{A^{\prime}}, M$ and by the assumption there is some stable formula $\delta(v, y) \in L^{\mathrm{eq}}$ and some tuple $a \in A^{\prime} M$ such that $\models \delta(e, a)$ and $\delta(v, a)$ forks over $A^{\prime}$. Let $p(v)=\operatorname{tp}_{\delta}(e / M)$ and let $c$ be its canonical base. Since $e \downarrow_{M} A^{\prime}$, the unique global $\delta$-type $\mathfrak{p}(v) \supseteq p(v)$ which is definable over $c$ extends $\operatorname{tp}_{\delta}\left(e / A^{\prime} M\right)$. Since $c$ is the canonical base of $\mathfrak{p}$ and $\mathfrak{p}$ forks over $A^{\prime}, c \notin \operatorname{acl}^{\mathrm{eq}}\left(A^{\prime}\right)$. It follows that $c \notin \operatorname{acl}^{\mathrm{eq}}(A)$. Hence $p(v)$ forks over $A$. Let $\varphi(v, b)$ be a finite conjunction of formulas of $p(v)$ which forks over $A$. Since $\varphi(v, b)$ is a conjunction of $\delta$-formulas, it is an instance of a stable formula. Moreover, $\models \varphi(e, b)$.

Corollary 2.3 $T$ has stable forking if and only if $T^{\mathrm{eq}}$ has stable forking. 
Proof: One direction follows from propositions 2.1 and 2.2. The rest is clear since $L^{\text {eq }}$ formulas with real free variables are equivalent to $L$-formulas.

\section{An example and some open problems}

We describe a theory $T$. Its language contains two binary relation symbols $E, F$, both are being interpreted as equivalence relations on the universe with some specific cross-cutting. The equivalence relation $E$ has infinitely many classes, all infinite. On the other hand $F$ has exactly one class of size $n$ for every $n \geq 1$, say consisting in the elements $a_{1}^{n}, \ldots, a_{n}^{n}$. For each $k \geq 1$, the elements $a_{k}^{k}, a_{k}^{k+1}, \ldots$ build an $E$-class. With these specifications, the set $\left\{a_{k}^{n} \mid 1 \leq k \leq n<\omega\right\}$ is the universe of a model $M$ of $T$. Note that $\operatorname{dcl}(\emptyset)=M$. The formula $E(x, y)$ has non finite cover property and $F(x, y)$ is stable. But $\exists y(E(x, y) \wedge F(y, z))$ is unstable, as witnessed by the sequences $\left(a_{i}^{i} \mid i \geq 1\right)$ and $\left(a_{1}^{j} \mid j \geq 1\right)$. This answers a question of M. C. Laskowski: Lemma 1.2 can not be generalized to the case where $\theta(v, x)$ is a non finite cover property formula. On the other hand, it shows that the proof of Proposition 2.1 can not be carried out trying to prove that the formula $\exists x\left(\pi_{E}(x)=\right.$ $v \wedge \delta(x, y)$ ) is stable (where $\pi_{E}$ is the mapping sending each tuple to its $E$-equivalence class). Since $T$ is interpretable in Presburger arithmetic, it is dp-minimal. But $T$ has the strict order property, hence it is not simple. This can be checked observing that the $E$-class of $a_{11}$ is infinite and has a definable linear ordering. It would be interesting to find a similar example in a simple theory $T$.

A. Chernikov has raised the question of whether Corollary 2.3 can be generalized to dependent forking. See [2] for the relevant definitions.

Let us finally mention a connected question asked by M. Ziegler. Assume all 1-types in $T$ have stable forking. Does it follow that $T$ has stable forking? A positive answer would be very helpful.

\section{References}

[1] E. Casanovas. Simple theories and hyperimaginaries, volume 39 of Lecture Notes in Logic. Cambridge University Press, 2011.

[2] A. Chernikov. Theories without the tree property of the second kind. Annals of Pure and Applied Logic, 165:695-723, 2014.

[3] B. Kim. Simplicity, and stability in there. The Journal of Symbolic Logic, 66:822-836, 2001.

[4] B. Kim and A. Pillay. Around stable forking. Fundamenta Mathematicae, 170:107-118, 2001.

[5] D. Palacín and F. O. Wagner. Elimination of hyperimaginaries and stable independence in simple CM-trivial theories. Notre Dame Journal of Formal Logic, 54:541-551, 2013.

[6] A. Peretz. Geometry of forking in simple theories. The Journal of Symbolic Logic, 71:347-359, 2006.

[7] A. Pillay. Geometric Stability Theory. Oxford University Press, 1996. 